\title{
19. INTERSTITIAL WATER STUDIES ON SMALL CORE SAMPLES, LEG $4^{1}$
}

\author{
F. L. Sayles, Woods Hole Oceanographic Institution, Woods Hole, Massachusetts, \\ F. T. Manheim, ${ }^{2}$ U. S. Geological Survey, Woods Hole, Massachusetts, and \\ K. M. Chan, California State College, Long Beach, California
}

\begin{abstract}
Reorganization and recodification of shipboard procedures for collecting interstitial waters has resulted in improved and more regular collection and analysis of pore fluids.

Comparative studies of waters squeezed and analyzed on shipboard and analyzed in the shore laboratory show generally good agreement, except for some aberrations whose sources are hard to track down. Influences of pressure and temperature during squeezing on composition of effluents were re-examined for clayey samples from Leg 4 cores. Pressure was not found to be significant, whereas the temperature effects are significant, but are less than variations attributable to diagenetic reactions in the sediments.

Conservative constituents, such as, chloride, sodium and bromide, remain relatively constant (within about 1.5 per cent) with changing depth in the holes; but, large depletions with respect to normal sea water occur in calcium (to $0.06 \mathrm{~g} / \mathrm{kg}$ ), magnesium (to 0.7 ), potassium (to 0.20 ), and sulfate (to 0.11 ) in most of the cores. On the other hand, large enrichments of calcium (to $1.57 \mathrm{~g} / \mathrm{kg}$ ) and lithium (to $1.7 \mathrm{ppm}$ ) occurred in Holes 24 and 24A. The depletion of several constituents in pore waters of Hole 26 (Vema fracture zone) caused a drop in total salt content to as low as $\mathbf{3 1} \mathrm{o} / \mathrm{oo}$. However, no real dilution effects are involved, since chloride and sodium values remain typical of those in ocean bottom waters.
\end{abstract}

\section{INTRODUCTION}

On Leg 4, shipboard pore water procedures were reorganized and recodified. This assisted in a more regular collection of assigned samples, both for the Woods Hole and UCLA groups, and it provided for the first extensive measurements of labile and gaseous constituents for the UCLA group. Except for salinity by index of refraction, this report will deal solely with the inorganic constituents in the pore waters. These constituents were determined in the Woods Hole laboratory on waters which were squeezed on board ship and preserved by heat-sealing in polyethylene pipes. Results from special samples which were separated from this set for isotopic determination will be reported on later by Irving Friedman, U. S. Geological Survey, Denver, Colorado.

\footnotetext{
${ }^{1}$ Contribution No. 2446 of the Woods Hole Oceanographic Institution.

${ }^{2}$ Publication approved by the Director, U.S. Geological Survey.
}

Several types of checks on sources of error in the data were made. Salinities determined on board ship were compared with those determined months later in the laboratory on aliquots heat-sealed in polyethylene pipe. Chlorides from the sealed fluids and from packaged sediments squeezed in the laboratory were also compared. Squeezing experiments (described in more detail in the report of F. L. Sayles) revealed that pressure effects did not exert significant influence on the salt content of total fluid volumes extracted from very clayey samples. Temperature of squeezing, however, did influence the composition of several constituents (Mangelsdorf et al., 1969; Bischoff et al., 1970), although the influences are considerably smaller than those evidently due to diagenetic interaction with the sediments.

In the pore water work, it is not possible to go back and resample cores, should handling problems be suspected. Where fluid quantities are limited to a few milliliters (as in this study), one can rarely perform replicate determinations to check out aberrant values. The small size of aliquots does not necessarily result in poorer 
optimum precision in analyses or transfer, but the errors or inadvertencies that do occur may be larger in magnitude than the errors involved in macro samples. It is therefore necessary to live with inaccuracies and aberrations that crop up from time to time and which the authors are painfully aware probably exceed natural variations in the analyzed constituents. The work so far has suggested, however, that most errors due to sampling or analysis occur randomly, whereas true variability in composition usually shows coherent trends within a given core or lithologic sequence. An exception to these relationships is the effect of temperature during squeezing, which will be dealt with separately in the discussion.

\section{METHODS}

Shipboard techniques are described in detail in the Initial Core Description Manual of the Deep Sea Drilling Project. Analytical techniques are given in detail in a paper in preparation (F. T. Manheim and K. M. Chan). A brief outline of methods is provided in the following section.

Samples were squeezed in a large stainless-steel apparatus (about a 9-centimeter piston diameter, modified from that described by Manheim, 1966) by a hydraulic press. Effluents, squeezed through filter paper and received in plastic disposable syringes, are passed through a micropore $(0.45 \mu)$ filter and heat-sealed in polyethylene pipe. A few drops are reserved for determination of salinity by means of a temperature-compensated hand refractometer. Care is always taken to use only internal portions of the cores for squeezing and to reject those portions of cores which are clearly disturbed or where the possibility of contamination by drilling fluid (sea water) is great. Parallel samples of core are also packed in glass jars with special (Polyseal $^{R}$ ) caps, normally with moistened, plastic-wrapped laboratory tissue inside to maintain 100 per cent moisture and take up condensation space. No moistened tissue touches the sediment itself.

For the inorganic constituents in the pore water, the following techniques were employed: chloride by Mohr titration with silver nitrate, using a weight burette; sulfate by microgravimetry; alkalinity by acid addition and back titration with standard base, using a mixed indicator;lithium, strontium, barium, boron and silicon by direct-reading emission spectrometry (solution spark); and silicon and bromide by colorimetry. Copenhagen standard sea water was used as the primary standard for calcium, potassium, sodium, magnesium, sulfate, chloride and served as a check on accuracy for boron, strontium and lithium determinations; major element values are taken from Culkin, 1965; estimated precision of analysis is indicated by the rounding of the results. The most accurate determination, intended as a standard of comparison for the others, is chloride (or rather, sum of chloride and bromide calculated as chloride). Copenhagen sea water is the titration standard, and special methods are employed to correct for differences in ionic strength; reproducibility is equal to $0.1 \mathrm{o} / \mathrm{oo}$ in terms of a standard deviation. Most of the discrepancies in balance between anions and cations arise from the sodium determination by atomic absorption (about 2 to 3 per cent reproducibility); however, ammonia could be high in some samples with abnormal alkalinity, and other errors may also play a role.

Accuracy in refractometry has improved. The authors note that if one waits for equilibration (30 to $45 \mathrm{sec}$ onds) and takes care in interpolating the light-dark interface with the reference scale engraved on the Goldberg Refractometer, a reproducibility equivalent to about $0.1 \mathrm{o} / \mathrm{oo}$ can be achieved; this is better than the 0.3 to $0.5 \mathrm{o} / \mathrm{oo}$ claimed by the manufacturer and achieved in use in Leg 1.

Currently under investigation is the influence which laboratory filter paper may exert on the analyzed constituents. Normally, paper is not considered a contaminant, but in this squeezing process a relatively small volume of fluid is passed through a relatively large filtering area (three 9-centimeter filters of usual high strength, high retentivity laboratory paper) for 2 to 10 milliliters of fluid.

Finally, new data (Bischoff et al., 1970) and current experiments at Woods Hole have confirmed the influence of temperature during the squeezing process on the composition of effluents (Mangelsdorf et al., 1969). These experiments suggest that because of the warming of clayey sediment samples to room temperature before squeezing, potassium concentrations are raised, and magnesium concentrations are lowered by as much as 0.05 and $0.15 \mathrm{~g} / \mathrm{kg}$, respectively. The results of Bischoff et al., (1970) indicate that even chloride can be influenced by temperature. No simple correction can be applied, because the Deep Sea Drilling Project samples are obtained from a sufficient depth to involve substantial variation in temperature, and no information on deep thermal gradient is available for the Atlantic legs. At the moment, the authors only plan to make spot checks on the temperature effect to test its influence. Insofar as the effect is evidently caused by ion-exchange properties of the clays, sediments with large non-clay components will be proportionately less affected.

\section{RESULTS}

Comparisons of determinations of salinity and chlorinity made aboard ship and in the laboratory are shown in Table 1. The later salinity determinations average 
TABLE 1

Comparative Results on Fluid Squeezed on Board Ship and Fluids Extracted in Laboratory on Stored Sediment Samples ${ }^{\mathrm{a}}$

\begin{tabular}{|c|c|c|c|c|c|c|c|c|c|c|c|}
\hline \multirow{2}{*}{\multicolumn{2}{|c|}{ Sample Designation }} & \multirow{3}{*}{$\begin{array}{c}\begin{array}{c}\text { Depth Below } \\
\text { Sea Bed } \\
(\mathrm{m})\end{array} \\
2\end{array}$} & \multirow[b]{2}{*}{ Description } & \multicolumn{4}{|c|}{ Chlorinity $(0 / 00)$} & \multirow[b]{2}{*}{$\mathrm{H}_{2} \mathrm{O}(\%)$} & \multirow[b]{2}{*}{ Ion Sum } & \multicolumn{2}{|c|}{ Salinity $(0 / 00)$} \\
\hline & & & & \multicolumn{2}{|c|}{ Ship-squeezed } & \multicolumn{2}{|c|}{ Laboratory-squeezed } & & & Refr. A & Refr. B \\
\hline \multirow[t]{3}{*}{ Hole 23} & $1-1$ & & Clay, olive & 19.6 & & - & & 48.9 & 35.8 & 36.3 & 35.4 \\
\hline & $3-2$ & 66 & "Red Clay," silty & 19.2 & & - & & 39.7 & 34.5 & 34.7 & 34.4 \\
\hline & $4-3$ & 120 & Clay, olive & 19.2 & & - & & 39.5 & 34.5 & 34.4 & 34.4 \\
\hline \multirow[t]{2}{*}{ Hole 24} & $2-1$ & 207 & Clay, gray, silty & 21.2 & & - & & 8.8 & - & - & 38.0 \\
\hline & $4-3$ & 233 & Clay-mudstone & 18.9 & & - & & 30.9 & 33.8 & 33.3 & 33.4 \\
\hline \multirow[t]{3}{*}{ Hole 26} & $1-3$ & 103 & Clay, silty & - & 19.4 & & 19.4 & 31.6 & - & 31.9 & 31.9 \\
\hline & $3-2$ & 230 & Clay, silty & 19.1 & & - & & 26.1 & 31.1 & 31.1 & 31.2 \\
\hline & $5-3$ & 482 & Clay, olive, calcareous & 19.2 & & - & & 22.3 & 32.1 & 31.6 & 31.9 \\
\hline \multirow[t]{4}{*}{ Hole 27} & $2-3$ & 147 & Clay, silty & 19.1 & & - & & 36.9 & 33.9 & 34.1 & 34.4 \\
\hline & $3-2$ & 239 & Clay, olive & 19.3 & & - & & 30.8 & 33.5 & 33.0 & 33.9 \\
\hline & $4-1$ & 246 & Clay, olive & 19.6 & & - & & 28.4 & 33.4 & 33.4 & 34.1 \\
\hline & $5-2$ & 374 & Clay, calcareous & 20.2 & & - & & 25.3 & 33.7 & 34.1 & 34.8 \\
\hline \multirow[t]{5}{*}{ Hole 27A } & $1-5$ & 34 & Clay, brown & 19.6 & 19.6 & & 19.6 & 42.7 & 35.6 & 34.7 & 35.5 \\
\hline & $2-3$ & 49 & Clay, brown, plastic & 19.4 & 20.0 & & 20.0 & 37.2 & 34.9 & 34.7 & 35.2 \\
\hline & $3-3$ & 58 & Clay, plastic & 19.5 & & - & & 37.5 & 35.2 & 34.1 & 35.2 \\
\hline & $4-3$ & 66 & Clay, brown, plastic & - & 19.8 & & 19.9 & 37.2 & - & 34.7 & 35.2 \\
\hline & $4-3$ & 66 & Clay, brown, plastic & - & & - & & 27.9 & - & 34.7 & 35.3 \\
\hline \multirow[t]{2}{*}{ Hole 28} & $2-3$ & 77 & Clay, varicolored & 19.2 & & - & & 41.4 & 34.5 & 34.7 & 35.0 \\
\hline & $3-1$ & 171 & Ooze, nannofossil & 19.5 & 19.4 & & 19.4 & 45.0 & 34.9 & 34.7 & 35.1 \\
\hline \multirow[t]{6}{*}{ Hole 29} & $1-3$ & 8 & Clay-ooze, gray-green & 19.6 & & - & & 34.2 & 35.8 & 35.2 & 35.5 \\
\hline & $4-3$ & 35 & Clay-ooze, olive & 19.6 & & - & & 39.0 & 35.3 & 34.4 & 35.4 \\
\hline & $9-2$ & 140 & Radiolarian ooze \& chert & 19.6 & 19.6 & & 19.6 & 61.7 & 35.2 & 34.9 & 35.8 \\
\hline & $12-6$ & (171) & Radiolarian ooze & 19.6 & 19.5 & & 19.6 & 67.8 & 35.1 & 34.7 & 35.3 \\
\hline & 14-3 & 192 & Radiolarian ooze & 19.6 & 19.7 & & 19.6 & 66.5 & $(35.1)$ & 34.7 & 35.4 \\
\hline & $17-1$ & 213 & Radiolarian ooze & 19.7 & & - & & 67.2 & 35.4 & 35.2 & 35.7 \\
\hline \multirow[t]{4}{*}{ Hole 29B } & $1-6$ & 65 & Clay, brown & 19.5 & & - & & 40.8 & 34.9 & 34.7 & 35.2 \\
\hline & $4-4$ & 96 & Clay, brown & 19.2 & & - & & 40.0 & 34.2 & 34.1 & 34.7 \\
\hline & $8-2$ & 126 & Radiolarian ooze + pumice & 19.6 & & - & & 30.1 & 35.5 & 34.7 & 35.6 \\
\hline & $9-4$ & 225 & Radiolarian ooze + pumice & 19.7 & & - & & 64.6 & 35.2 & 35.2 & 35.8 \\
\hline \multirow[t]{2}{*}{ Hole 30} & $2-4$ & 67 & Clay-ooze, gray-green & 19.8 & & - & & 41.9 & 33.2 & 32.7 & 33.0 \\
\hline & $3-3$ & 115 & Clay-ooze, gray-green & 19.7 & & - & & 35.7 & 32.9 & 31.9 & 32.6 \\
\hline
\end{tabular}


TABLE 1 - Continued

\begin{tabular}{|c|c|c|c|c|c|c|c|c|c|c|c|}
\hline \multirow{2}{*}{\multicolumn{2}{|c|}{ Sample Designation }} & \multirow{2}{*}{$\begin{array}{l}\text { Depth Below } \\
\text { Sea Bed } \\
\text { (m) }\end{array}$} & \multirow[b]{2}{*}{ Description } & \multicolumn{4}{|c|}{ Chlorinity (o/oo) } & \multirow[b]{2}{*}{$\mathrm{H}_{2} \mathrm{O}(\%)$} & \multirow[b]{2}{*}{ Ion Sum } & \multicolumn{2}{|c|}{ Salinity (o/oo) } \\
\hline & & & & \multicolumn{2}{|c|}{ Ship-squeezed } & \multicolumn{2}{|c|}{ Laboratory-squeezed } & & & Refr. A & Refr. B \\
\hline \multicolumn{12}{|c|}{ Hole 30-Continued } \\
\hline & $6-2$ & 176 & Siltstone, clayey & 19.6 & & - & & 31.4 & 31.6 & 31.6 & 31.6 \\
\hline & $11-2$ & 388 & Siltstone, clayey & 19.8 & & - & & 30.8 & 33.0 & 31.9 & 32.6 \\
\hline & $13-1$ & 403 & Siltstone, clayey & 19.7 & & - & & 33.7 & 32.9 & - & 32.7 \\
\hline & $15-4$ & 419 & Siltstone, clayey & 19.8 & 19.2 & $(! ?)$ & 19.2 & 33.5 & 33.2 & 32.7 & 33.0 \\
\hline \multirow[t]{3}{*}{ Hole 31} & $3-6$ & 68 & Marl, gray & 19.6 & & - & & 38.8 & 35.4 & 34.4 & 34.6 \\
\hline & $9-2$ & 216 & Chalk, light gray & 19.9 & & - & & 33.6 & 35.3 & 35.2 & 35.2 \\
\hline & $10-2$ & 274 & Chalk, nannoplankton, compact & 20.0 & 19.5 & & 19.6 & 32.2 & 35.3 & 35.2 & 35.3 \\
\hline
\end{tabular}

${ }^{a}$ See text for details of preservation. "A" refers to samples squeezed, filtered $(0.45 \mu)$ and checked by refractometry aboard ship, whereas "B" refers to similar samples determined in the laboratory. Note that the salinity estimated by refractive index on board ship has not been corrected for zero adjustment of the refractometer. This will probably raise the salinity by a few tenths of a $\mathrm{g} / \mathrm{kg}$; the laboratory values have been corrected. Calibration is for standard sea water according to Behrens (1965). The samples squeezed in the laboratory do not come from the same core section as those squeezed on board ship but are usually separated by less than 10 centimeters. Ion sum includes $\mathrm{Na}, \mathrm{K}, \mathrm{Ca}, \mathrm{Mg}, \mathrm{Cl}, \mathrm{SO}_{4}, \mathrm{HCO}_{3}$ (calculated from alkalinity). It does not include minor constituents or $\mathrm{NH}_{4}$ which could be fairly large in some samples. $\mathrm{H}_{2} \mathrm{O}(\%)$ refers to water content determined by drying at $110-120^{\circ} \mathrm{C}$. 
TABLE 2

Major Inorganic Constituents of Pore Waters Values Reported in $\mathrm{g} / \mathrm{kg}(\mathrm{o} / \mathrm{oo})$ Unless Otherwise Indicated

\begin{tabular}{|c|c|c|c|c|c|c|c|c|c|c|c|c|c|c|}
\hline Sample Designation & $\begin{array}{l}\text { Depth } \\
(\mathrm{m})\end{array}$ & Description & Age & $\mathrm{Na}$ & $\mathbf{K}$ & $\mathrm{Ca}$ & $\mathrm{Mg}$ & $\begin{array}{l}\Sigma \text { Cations } \\
(\mathrm{meq} / \mathrm{kg})\end{array}$ & $\mathrm{Cl}$ & $\mathrm{SO}_{4}$ & $\begin{array}{c}\text { Alk } \\
(\mathrm{meq} / \mathrm{kg})\end{array}$ & $\mathrm{HCO}_{3}^{\mathrm{b}}$ & $\begin{array}{l}\text { ¿Anions } \\
(\mathrm{meq} / \mathrm{kg})\end{array}$ & Sum \\
\hline \multicolumn{15}{|c|}{ Hole $23\left(6^{\circ} 08.7^{\prime} \mathrm{S}, 31^{\circ} 02.6^{\prime} \mathrm{W}, 5079\right.$ meters $)$} \\
\hline $1-1 \quad(130-145)$ & 2 & Clay, olive gray & Pleistocene & 11.1 & 0.49 & 0.47 & 1.18 & 615 & 19.6 & $(2.7)^{\mathrm{a}}$ & 3.17 & 0.19 & $(615)$ & 35.8 \\
\hline $1-1 \quad(130-145)$ & 2 & Clay, olive gray & Pleistocene & 11.1 & 0.49 & 0.47 & 1.18 & 615 & 19.6 & $(2.7)^{\mathrm{a}}$ & 3.17 & 0.19 & $(615)$ & 35.8 \\
\hline $3-2 \quad(130-145)$ & 66 & $\begin{array}{l}\text { "Red clay" silty, } \\
\text { zeolitic, Mn. pellets }\end{array}$ & Miocene & 10.6 & 0.34 & 0.49 & 1.19 & 592 & 19.2 & 2.45 & 3.99 & 0.24 & 596 & 34.5 \\
\hline $4-3 \quad(54-60)$ & 120 & Clay, olive, green, $\mathrm{Mn}$ & L. Miocene & (10.6) & 0.33 & 0.60 & 1.16 & $(592)$ & 19.2 & 2.45 & 3.16 & 0.19 & 596 & 34.5 \\
\hline \multicolumn{15}{|c|}{ Hole $24\left(6^{\circ} 16.3^{\prime} \mathrm{S}, 30^{\circ} 53.5^{\prime} \mathrm{W}, 5142\right.$ meters $)$} \\
\hline $2-1 \quad(62-72)$ & 207 & $\begin{array}{l}\text { Clay, gray-green } \\
\text { silty-sandy, glauconite }\end{array}$ & L. Miocene & 11.1 & 0.35 & 0.82 & 1.13 & 625 & 21.2 & 1.83 & - & - & - & - \\
\hline $3-4 \quad(142-146)$ & 233 & $\begin{array}{l}\text { Clay-mudstone. glauconite } \\
\text { zeolitic }\end{array}$ & L. Miocene & 10.3 & 0.22 & 0.86 & 1.06 & 583 & 18.9 & $(2.2)^{\mathrm{a}}$ & 4.84 & 0.29 & $(583)$ & 33.8 \\
\hline \multicolumn{15}{|c|}{ Hole $24 \mathrm{~A}\left(6^{\circ} 16.6^{\prime} \mathrm{S}, 30^{\circ} 53.5^{\prime} \mathrm{W}, 5242\right.$ meters $)$} \\
\hline $3-1 \quad(122-131)$ & 542 & Graywacke, dk. brown & Paleocene-Cret. & 10.5 & 0.26 & 1.57 & 0.83 & 610 & 20.6 & $(1.3)^{\mathrm{a}}$ & 2.97 & 0.18 & $(610)$ & 35.2 \\
\hline \multicolumn{15}{|c|}{ Hole $26\left(10^{\circ} 53.6^{\prime} \mathrm{N}, 44^{\circ} 02.6^{\prime} \mathrm{W}, 5160\right.$ meters; Vema fracture zone) } \\
\hline$(0-6)$ & 103 & Clay, dk. olive, silty & Pleistocene & 10.2 & 0.30 & 0.23 & 1.04 & 548 & 19.3 & 1.24 & 10.5 & 0.64 & 566 & 32.9 \\
\hline$(6-12)$ & 230 & Clay, olive-gray, silty & Pleistocene & 10.3 & 0.24 & 0.24 & 0.81 & 534 & 19.1 & 0.06 & 4.71 & 0.29 & 545 & 31.1 \\
\hline$(4-9)$ & 482 & Clay, olive-gray, calcareous & Pleistocene & 10.9 & 0.16 & 0.47 & 0.71 & 559 & 19.2 & 0.49 & 3.13 & 0.19 & 555 & 32.1 \\
\hline \multicolumn{15}{|c|}{ Hole $27\left(15^{\circ} 51.4^{\prime} \mathrm{S}, 56^{\circ} 52.8^{\prime} \mathrm{W}, 5251\right.$ meters $)$} \\
\hline$(0-5)$ & 147 & $\begin{array}{l}\text { Clay, gray, silty } \\
\text { sandy, glauconite pyrite }\end{array}$ & Miocene & 10.9 & 0.23 & 0.83 & 1.04 & 607 & 19.1 & 1.66 & 2.77 & 0.17 & 576 & 33.2 \\
\hline$(0-5)$ & 239 & $\begin{array}{l}\text { Clay, olive, brown, gc., } \\
\text { glauconite }\end{array}$ & Miocene & 10.7 & 0.21 & 0.99 & 0.99 & 601 & 19.3 & 1.07 & 4.31 & 0.26 & 571 & 32.8 \\
\hline $4-1 \quad(140-145)$ & 246 & $\begin{array}{l}\text { Clay, olive brown, glauconite } \\
\text { pyrite }\end{array}$ & Miocene & 10.3 & 0.22 & 1.05 & 0.98 & 587 & 19.6 & 1.00 & 3.41 & 0.21 & 577 & 33.2 \\
\hline $5-2 \quad(141-145)$ & 374 & Clay, gray, calcareous & Oligocene & 10.7 & 0.20 & 1.21 & 0.83 & 549 & 20.2 & 0.46 & 1.48 & 0.09 & 580 & 33.7 \\
\hline \multicolumn{15}{|c|}{ Hole $27 \mathrm{~A}\left(15^{\circ} 51.4^{\prime} \mathrm{S}, 56^{\circ} 52.8^{\prime} \mathrm{W}, 5245\right.$ meters $)$} \\
\hline $1-5 \quad(139-145)$ & 34 & $\begin{array}{l}\text { Clay, brown, sticky, } \\
\text { non-calcareous }\end{array}$ & Pleistocene & 11.1 & 0.40 & 0.55 & 1.13 & 613 & 19.6 & 2.51 & 4.50 & 0.28 & 609 & 35.6 \\
\hline
\end{tabular}


Table 2 - Continued

\begin{tabular}{|c|c|c|c|c|c|c|c|c|c|c|c|c|c|c|}
\hline Sample Designation & $\begin{array}{c}\text { Depth } \\
(\mathrm{m})\end{array}$ & Description & Age & $\mathrm{Na}$ & $\mathbf{K}$ & $\mathrm{Ca}$ & $\mathrm{Mg}$ & $\begin{array}{l}\Sigma \text { Cations } \\
(\mathrm{meq} / \mathrm{kg})\end{array}$ & $\mathrm{Cl}$ & $\mathrm{SO}_{4}$ & $\begin{array}{c}\text { Alk } \\
(\mathrm{meq} / \mathrm{kg})\end{array}$ & $\mathrm{HCO}_{3}^{\mathrm{b}}$ & $\begin{array}{l}\sum \text { Anions } \\
(\mathrm{meq} / \mathrm{kg})\end{array}$ & Sum \\
\hline \multicolumn{15}{|c|}{ Hole $27 \mathrm{~A}\left(15^{\circ} 51.4^{\prime} \mathrm{S}, 56^{\circ} 52.8^{\prime} \mathrm{W}, 5245\right.$ meters) (Continued) } \\
\hline $2-3 \quad(127-134)$ & 49 & Clay, gray-brown, plastic & Pleistocene & 10.9 & 0.35 & 0.60 & 1.12 & 604 & 19.4 & 2.24 & 5.21 & 0.32 & 598 & 34.9 \\
\hline $3-3 \quad(130-134)$ & 58 & Clay, brown, plastic & Pleistocene & 11.1 & 0.37 & 0.60 & 1.08 & 611 & 19.5 & 2.19 & 5.56 & 0.34 & 602 & 35.2 \\
\hline $4-3 \quad(33-37)$ & 66 & Clay, brown, plastic & Pleistocene & 11.0 & 0.36 & 0.60 & 1.07 & 605 & - & $(3.28)^{\mathrm{a}}$ & 5.08 & 0.31 & - & - \\
\hline $4-3 \quad(70-86)$ & 66 & Clay, brown, plastic & Pleistocene & - & - & - & 1.08 & - & - & - & - & - & - & - \\
\hline \multicolumn{15}{|c|}{ Hole $28\left(20^{\circ} 35.2^{\prime} \mathrm{N}, 65^{\circ} 37.3^{\prime} \mathrm{W}, 5521\right.$ meters; Puerto Rico outer ridge) } \\
\hline $2-3 \quad(140-145)$ & 77 & Clay, varicolored & $?$ & 10.6 & 0.34 & 0.71 & 1.08 & 593 & 19.2 & 2.32 & 3.76 & 0.23 & 594 & 34.5 \\
\hline $3-1 \quad(140-145)$ & 171 & OOze, nannofossil & Eocene & 10.9 & 0.35 & 0.89 & 1.06 & 614 & 19.5 & 1.96 & 3.68 & 0.22 & 594 & 34.9 \\
\hline \multicolumn{15}{|c|}{ Hole $29\left(14^{\circ} 47.1^{\prime} \mathrm{N}, 69^{\circ} 19.4^{\prime} \mathrm{W}, 4247\right.$ meters; Caribbean Sea $)$} \\
\hline $1-3 \quad(140-145)$ & 8 & Clay-ooze, gray-green & $\begin{array}{l}\text { Recent } \\
\text { Pleistocene }\end{array}$ & 11.4 & 0.44 & 0.47 & 1.18 & 628 & 19.6 & 2.56 & 2.57 & 0.16 & 611 & 35.8 \\
\hline $4-3 \quad(140-145)$ & 35 & Clay-ooze, olive & Pliocene & 11.0 & 0.41 & 0.50 & 1.13 & 607 & 19.6 & 2.47 & 2.24 & 0.14 & 608 & 35.3 \\
\hline $9-2 \quad(140-145)$ & 140 & $\begin{array}{l}\text { Radiolarian ooze \& } \\
\text { chert }\end{array}$ & M. Eocene & 10.9 & 0.37 & 0.70 & 1.12 & 611 & 19.6 & 2.37 & 2.52 & 0.15 & 607 & 35.2 \\
\hline $12-6 \quad(143-148)$ & (171) & Radiolarian ooze & M. Eocene & 11.1 & 0.36 & 0.62 & 1.12 & 618 & 19.6 & 1.99 & 2.70 & 0.16 & 600 & 35.1 \\
\hline $14-3 \quad(135-140)$ & 192 & Radiolarian ooze & M. Eocene & 11.1 & 0.36 & 0.73 & 1.14 & 622 & 19.6 & $(2.0)^{\mathrm{a}}$ & 2.96 & 0.18 & - & - \\
\hline $17-1 \quad(136-143)$ & 213 & $\begin{array}{l}\text { Radiolarite, cherty } \\
\text { dolomite }\end{array}$ & M. Eocene & 11.1 & 0.34 & 0.78 & 1.13 & 624 & 19.7 & 2.16 & 2.88 & 0.18 & 605 & 35.4 \\
\hline \multicolumn{15}{|c|}{ Hole 29B $\left(14^{\circ} 47.1^{\prime} \mathrm{N}, 69^{\circ} 19.4^{\prime} \mathrm{W}, 4247\right.$ meters; Caribbean Sea $)$} \\
\hline $1-6 \quad(139-145)$ & 65 & Clay, brown & M. Miocene & 10.8 & 0.34 & 0.60 & 1.13 & 602 & 19.5 & 2.42 & 2.36 & 0.14 & 604 & 35.0 \\
\hline $4-4 \quad(48-53)$ & 96 & Clay, brown & M. Miocene & 10.6 & 0.31 & 0.64 & 1.14 & 595 & 19.2 & 2.29 & 1.98 & 0.12 & 594 & 34.4 \\
\hline $8-2 \quad(70-77)$ & 126 & $\begin{array}{l}\text { Radiolarian ooze \& } \\
\text { pumice }\end{array}$ & U. Eocene & 11.1 & 0.43 & 0.69 & 1.14 & 622 & 19.6 & 2.36 & 2.82 & 0.17 & 606 & 35.6 \\
\hline $9-4 \quad(71-77)$ & 225 & $\begin{array}{l}\text { Radiolarian ooze \& } \\
\text { pumice }\end{array}$ & M. Eocene & 10.9 & 0.35 & 0.69 & 1.13 & 611 & 19.7 & 2.24 & 2.13 & 0.13 & 606 & 35.2 \\
\hline \multicolumn{15}{|c|}{ Hole $30\left(12^{\circ} 52.9^{\prime} \mathrm{N}, 63^{\circ} 23.0^{\prime} \mathrm{W}, 1218\right.$ meters; Aves Ridge $)$} \\
\hline $2-4 \quad(131-137 ?)$ & 67 & Clay-ooze, gray-green & R.-Pleist. & 11.1 & 0.38 & 0.06 & 0.98 & 578 & 19.8 & 0.33 & 9.59 & 0.58 & 576 & 33.3 \\
\hline $3-3 \quad(144-150)$ & 115 & Clay-ooze, gray-green & R.-Pleist. & 11.1 & 0.42 & 0.14 & 0.85 & 571 & 19.7 & 0.23 & 7.96 & 0.49 & 571 & 33.0 \\
\hline 6-2 (139-144) & 176 & Siltstone, clayey & Pleistocene & 10.7 & 0.28 & 0.21 & 0.58 & 562 & 19.6 & 0.11 & 2.35 & 0.14 & 565 & 31.7 \\
\hline $11-2 \quad(41-73)$ & 388 & Siltstone, clayey & M. Miocene & 11.1 & 0.25 & 0.58 & 0.74 & 579 & 19.8 & 0.38 & 1.57 & 0.09 & 570 & 32.9 \\
\hline $13-1 \quad(148)$ & 403 & Siltstone, clayey & M. Miocene & 10.9 & 0.23 & 0.63 & 0.76 & 574 & 19.7 & 0.56 & 1.33 & 0.08 & 571 & 33.0 \\
\hline$(8-12)$ & 419 & Siltstone, clayey & M. Miocene & 11.1 & 0.28 & 0.58 & 0.79 & 584 & 19.8 & 0.53 & 1.73 & 0.11 & 574 & 33.3 \\
\hline
\end{tabular}


TABLE 2 - Continued

\begin{tabular}{|c|c|c|c|c|c|c|c|c|c|c|c|c|c|c|}
\hline Sample Designation & $\begin{array}{l}\text { Depth } \\
\text { (m) }\end{array}$ & Description & Age & $\mathrm{Na}$ & $\mathrm{K}$ & $\mathrm{Ca}$ & $\mathrm{Mg}$ & $\begin{array}{l}\Sigma \text { Cations } \\
(\mathrm{meq} / \mathrm{kg})\end{array}$ & $\mathrm{Cl}$ & $\mathrm{SO}_{4}$ & $\begin{array}{c}\text { Alk } \\
(\mathrm{meq} / \mathrm{kg})\end{array}$ & $\mathrm{HCO}_{3}^{\mathrm{b}}$ & $\begin{array}{l}\sum_{\text {Anions }} \\
(\mathrm{meq} / \mathrm{kg})\end{array}$ & Sum \\
\hline \multicolumn{15}{|c|}{ Hole $31\left(14^{\circ} 56.6^{\prime} \mathrm{N}, 72^{\circ} 06.6^{\prime} \mathrm{W}, 3363\right.$ meters $)$} \\
\hline $3-6$ & 68 & Marl, gray & U. Pliocene & 11.1 & 0.42 & 0.76 & 1.19 & 630 & 19.7 & 2.15 & 2.22 & 0.14 & 602 & 35.4 \\
\hline $9-2$ & 216 & Chalk, light gray & L. Miocene & 11.1 & 0.37 & 0.64 & 1.03 & 609 & 19.9 & 2.06 & 3.03 & 0.18 & 608 & 35.3 \\
\hline $10-2$ & 274 & $\begin{array}{l}\text { Chalk, nannoplankton } \\
\text { compact }\end{array}$ & L. Miocene & 11.0 & 0.34 & 0.68 & 0.96 & 600 & 20.0 & 2.13 & 2.84 & 0.17 & 613 & 35.4 \\
\hline
\end{tabular}

aSulfates in parentheses are calculated by difference, assuming difference between cations and anions is solely due to $\mathrm{SO}_{4}$. Anion sum in these cases is assumed equal to cations and is shown in parenthesis.

$\mathrm{bHCO}_{3}$ is calculated from alkalinity, assuming all alkalinity is due to this species. This assumption is not strictly correct, but is a fair approximation for the purposes of calculating the sum. 
TABLE 3

Minor Constituents of Pore Waters-All Values in $\mathrm{mg} / \mathrm{kg}(\mathrm{ppm})^{\mathrm{a}}$

\begin{tabular}{|c|c|c|c|c|c|c|c|c|}
\hline \multirow{2}{*}{ Sample } & \multirow[b]{2}{*}{$\begin{array}{l}\text { Depth } \\
\text { (m) }\end{array}$} & \multirow[b]{2}{*}{ Description } & \multirow[b]{2}{*}{ Age } & \multirow[b]{2}{*}{$\mathrm{Li}$} & \multicolumn{3}{|c|}{ PPM } & \multirow[b]{2}{*}{$\mathrm{Br}$} \\
\hline & & & & & B & $\mathrm{Sr}$ & $\mathrm{Ba}$ & \\
\hline \multicolumn{9}{|c|}{ Hole $23\left(6^{\circ} 08.7^{\prime} \mathrm{S}, 31^{\circ} 02.6^{\prime} \mathrm{W}, 5079\right.$ meters $)$} \\
\hline $1-1$ & 2 & Clay, olive gray & Pleistocene & 0.19 & 8 & 8.2 & 0.12 & - \\
\hline $3-2$ & 66 & $\begin{array}{l}\text { "Red clay" silty, zeolitic, Mn. } \\
\text { pellets }\end{array}$ & Miocene & 0.32 & 7 & 11.0 & 0.11 & - \\
\hline $4-3$ & 120 & Clay, olive-green, Mn. & L. Miocene & 0.26 & 7 & 9.0 & 0.09 & 66 \\
\hline \multicolumn{9}{|c|}{ Hole $24\left(6^{\circ} 16.3^{\prime} \mathrm{S}, 30^{\circ} 53.5^{\prime} \mathrm{W}, 5142\right.$ meters $)$} \\
\hline $2-1$ & 207 & Clay, gray-green, silty-sandy,glauconit & eL. Miocene & 1.50 & 4 & 31.0 & 0.17 & - \\
\hline $4-3$ & 233 & Clay-mudstone, glauconite, zeolitic & L. Miocene & 1.25 & 4 & 26.0 & 0.18 & - \\
\hline \multicolumn{9}{|c|}{ Hole $24 \mathrm{~A}\left(6^{\circ} 16.6^{\prime} \mathrm{S}, 30^{\circ} 53.5^{\prime} \mathrm{W}, 5142\right.$ meters $)$} \\
\hline $3-1$ & 542 & Graywacke, dark brown & $\begin{array}{l}\text { Paleocene- } \\
\text { Cret. }\end{array}$ & 1.70 & 3 & 20.0 & 0.19 & - \\
\hline \multicolumn{9}{|c|}{ Hole $26\left(10^{\circ} 53.6^{\prime} \mathrm{N}, 44^{\circ} 02.6^{\prime} \mathrm{W}, 5160\right.$ meters; Vema fracture zone $)$} \\
\hline $1-3$ & 103 & Clay, dark olive, silty & Pleistocene & 0.05 & 5 & 6.6 & 1.4 & - \\
\hline $3-2$ & 230 & Clay, olive-gray, silty & Pleistocene & 0.05 & 3 & 7.0 & 1.7 & - \\
\hline $5-3$ & 482 & Clay, olive-gray, calcareous & Pleistocene & 0.14 & 2 & 10.7 & 1.6 & - \\
\hline \multicolumn{9}{|c|}{ Hole $27\left(15^{\circ} 51.4^{\prime} \mathrm{S}, 56^{\circ} 52.8^{\prime} \mathrm{W}, 5251\right.$ meters $)$} \\
\hline $2-3$ & 147 & $\begin{array}{l}\text { Clay, gray, silty, sandy, } \\
\text { glauconite pyrite }\end{array}$ & Miocene & 0.40 & 5 & 9.3 & 0.17 & - \\
\hline $3-2$ & 239 & Clay, olive brown, glauconite, pyrite & Miocene & 0.47 & 4 & 12.0 & 0.19 & 68 \\
\hline $4-1$ & 246 & Clay, olive brown, glauconite, pyrite & Miocene & 0.43 & 4 & 12.0 & 0.17 & - \\
\hline $5-2$ & 374 & Clay, gray calcareous & Oligocene & 0.39 & 4 & 17.0 & 0.45 & - \\
\hline \multicolumn{9}{|c|}{ Hole $27 \mathrm{~A}\left(15^{\circ} 51.4^{\prime} \mathrm{S}, 56^{\circ} 52.8^{\prime} \mathrm{W}, 5245\right.$ meters $)$} \\
\hline $1-5$ & 34 & $\begin{array}{l}\text { Clay, brown, sticky, non- } \\
\text { calcareous }\end{array}$ & Pleistocene & 0.11 & 3 & 6.1 & 0.13 & 66 \\
\hline $2-3$ & 49 & Clay, gray-brown, plastic & Pleistocene & 0.12 & 3 & 6.3 & 0.08 & 67 \\
\hline $3-3$ & 58 & Clay, brown, plastic & Pleistocene & 0.13 & 3 & 6.7 & 0.08 & 70 \\
\hline $4-3$ & 66 & Clay, brown, plastic & Pleistocene & 0.12 & 3 & 6.6 & 0.09 & 68 \\
\hline $4-3$ & 66 & Clay, brown, plastic & Pleistocene & 0.13 & 3 & 5.7 & 0.16 & - \\
\hline \multicolumn{9}{|c|}{ Hole $28\left(20^{\circ} 35.2^{\prime} \mathrm{N}, 65^{\circ} 37.3^{\prime} \mathrm{W}, 5521\right.$ meters; Puerto Rico outer ridge $)$} \\
\hline $2-3$ & 77 & Clay, varicolored & $?$ & 0.63 & 5 & 8.8 & 0.10 & 67 \\
\hline $3-1$ & 171 & Ooze, nannofossil & Eocene & 1.26 & 3 & 12.7 & 0.14 & 67 \\
\hline \multicolumn{9}{|c|}{ Hole $29\left(14^{\circ} 47.1^{\prime} \mathrm{N}, 69^{\circ} 19.4^{\prime} \mathrm{W}, 4247\right.$ meters; Caribbean Sea $)$} \\
\hline $1-3$ & 8 & Clay-ooze, gray-green & Pleistocene & 0.18 & 6 & 7.7 & 0.07 & 67 \\
\hline 4-3 & 35 & Clay-ooze, olive & Pliocene & 0.16 & 5 & 7.0 & 0.08 & 67 \\
\hline
\end{tabular}


TABLE 3 - Continued

\begin{tabular}{|c|c|c|c|c|c|c|c|c|}
\hline Sample & $\begin{array}{l}\text { Depth } \\
\text { (m) }\end{array}$ & Description & Age & $\mathrm{Li}$ & B & PPM & $\mathrm{Ba}$ & $\mathrm{Br}$ \\
\hline \multicolumn{9}{|c|}{ Hole 29-Continued } \\
\hline $9-2$ & 140 & Radiolarian ooze + chert & M. Eocene & 0.27 & 3 & 9.4 & 0.05 & 68 \\
\hline $12-6$ & $(171)$ & Radiolarian ooze & M. Eocene & 0.27 & 4 & 10.0 & 0.05 & 68 \\
\hline $14-3$ & 192 & Radiolarian ooze & M. Eocene & 0.25 & 4 & 8.9 & 0.06 & 68 \\
\hline $17-1$ & 213 & Radiolarite, cherty dolomite & M. Eocene & 0.24 & 3 & 9.5 & 0.10 & 69 \\
\hline \multicolumn{9}{|c|}{ Hole $29 \mathrm{~B}\left(14^{\circ} 47.1^{\prime} \mathrm{N}, 69^{\circ} 19.4^{\prime} \mathrm{W}, 4247\right.$ meters; Caribbean $)$} \\
\hline $1-6$ & 65 & Clay, brown & M. Miocene & 0.19 & 6 & 7.8 & 0.11 & 69 \\
\hline $4-4$ & 96 & Clay, brown & M. Miocene & 0.26 & 7 & 9.6 & 0.13 & 68 \\
\hline $8-2$ & 126 & Radiolarian ooze + pumice & U. Eocene & 0.18 & 5 & 8.3 & 0.13 & 75 \\
\hline $9-4$ & 225 & Radiolarian ooze + pumice & M. Eocene & 0.32 & 3 & 9.3 & 0.07 & 69 \\
\hline \multicolumn{9}{|c|}{ Hole $30\left(12^{\circ} 52.9^{\prime} \mathrm{N}, 63^{\circ} 23.0^{\prime} \mathrm{W}, 1218\right.$ meters; Aves Ridge $)$} \\
\hline $2-4$ & 67 & Clay-ooze, gray-green & R.Pleist. & 0.19 & 4 & 6.4 & 0.16 & 68 \\
\hline $3-3$ & 115 & Clay-ooze, gray-green & R.-Pleist. & 0.14 & 3 & 7.8 & 4.2 & 76 \\
\hline $6-2$ & 176 & Siltstone, clayey & Pleistocene & 0.13 & 2 & 9.2 & 5.9 & 80 \\
\hline $11-2$ & 388 & Siltstone, clayey & M. Miocene & 0.34 & 2 & 22.0 & 5.4 & - \\
\hline $13-1$ & 403 & Siltstone, clayey & M. Miocene & 0.46 & 4 & 25.0 & 0.26 & 76 \\
\hline $15-4$ & 419 & Siltstone, clayey & M. Miocene & 0.47 & 4 & 25.0 & 0.19 & 74 \\
\hline \multicolumn{9}{|c|}{ Hole $31\left(14^{\circ} 56.6^{\prime} \mathrm{N}, 72^{\circ} 06.6^{\prime} \mathrm{W}, 3363\right.$ meters $)$} \\
\hline $3-6$ & 68 & Marl, gray & U. Pliocene & 0.43 & 4 & 49.0 & 0.21 & - \\
\hline $9-2$ & 216 & Chalk, light gray & L. Miocene & 0.21 & 5 & 15.0 & 0.35 & 74 \\
\hline $10-2$ & 274 & Chalk, nannoplankton compact & L. Miocene & 0.35 & 4 & 51.0 & 0.06 & 65 \\
\hline
\end{tabular}


about $0.5 \mathrm{o} / \mathrm{oo}$ greater than those determined on board ship. However, the shipboard refractometer has not been corrected for zero deviation. Zero is usually low on instruments received from the factory, and the shipboard values may well be low by the above amounts. Within the precision of the determinations and that of the summations, the salinity given by refractive index has agreed well with the ion sum (usually within $0.3 \mathrm{o} / \mathrm{oo}$ ).

The pooled variances from means of chlorinities of waters squeezed on board ship and in the laboratory have a standard deviation of about 0.2 o/oo, mainly due to a few aberrant values. Neither in these nor in previous comparative studies of samples squeezed aboard ship and in the laboratory do we see evidence for major systematic errors in chlorinity attributable to the storage of sediments, where proper precautions have been observed. Since the analytical scatter for chloride is equal to or normally less than $0.1 \mathrm{o} / \mathrm{oo}$, in terms of a standard deviation, the relatively large deviations in samples from Holes 30 and 31 must probably be sought somewhere in contamination, sampling, packaging or other manipulations preparatory to squeezing.

Finally, the average bottom water chlorinity for the western equatorial Atlantic is about $19.0 \mathrm{o} / \mathrm{oo}$, or slightly less (Muromtsev, 1963). Most of the interstitial chlorinity values are more than this. Although a part of the difference may be due to the handling of the sediments and pore waters (evaporation) or other artificially introduced factors, the possibility cannot be excluded that some of the excess chlorinity is real.

As in previous legs, the least changes in ionic ratios occurred in pelagic sediments such as oozes and "red clay." Such sediments were typical of Hole 29 and the upper part of Hole 23. A decrease in potassium values with depth and a moderate depletion in magnesium were similar to features seen in the pelagic sediments from Legs 2 and 3. By subtracting something less than 10 per cent potassium and adding between 1 to 2 per cent magnesium, to compensate for possible effects caused by raising sediment temperature to shipboard laboratory values before squeezing, one can arrive at modest excesses of potassium over oceanic water, and magnesium values roughly equivalent to standard oceanic concentration in the interstitial water from the top of Hole 23. However, in cores containing detrital clays and clastics which show affinities to land-derived products, drastic depletions in both potassium and magnesium are common. In Hole 26, for example, potassium dropped to only $0.16 \mathrm{~g} / \mathrm{kg}$ at 482 meters depth, compared with 0.38 for standard sea water; magnesium fell to $0.71 \mathrm{~g} / \mathrm{kg}$, compared with normal $1.27 \mathrm{~g} / \mathrm{kg}$. At the same time, sulfate at 230 meters in this hole fell to the lowest value yet reported in JOIDES pore water: $0.06 \mathrm{~g} / \mathrm{kg}$, compared with 2.70 for normal ocean water. Even calcium, lithium and strontium were depleted in the upper levels of the hole. The net effect (dominated by the loss of sulfate) is to drop total salt content to levels as low as $31.1 \mathrm{o} / \mathrm{oo}$, without a major drop in the "conservative" constituents, especially chloride.

Elsewhere in Leg 4, reconstitution of potassium and magnesium in sediments (assumed from sharp concentration drops in the pore water) does not preclude a major increase in calcium concentrations, which occurred in Holes 24A and 27.

Among the minor constituents, the authors note a general increase in lithium over that documented for oceanic waters, relatively minor changes in strontium, except where large increases in calcium were found, and generally sulfate-controlled levels of barium, implying that the barium sulfate solubility limits the concentration of this element. As usual, maximum fluctuations in alkalinity and depletions of sulfate occurred in those sediments with rapid accumulation rates and apparent access to terrigenous products.

Finally, some comment on silica analysis is needed. Total silica (Table 4) is determined by emission spectrometer (solution spark). In this technique both soluble and nonreactive or insoluble silica are analyzed since all silica, regardless of origin, is volatilized and excited in the high-temperature spark. That is, any silica which may have passed the micropore filtration step on board ship or may have settled out in colloidal suspension subsequently would be included, whereas such phases would not be detected by the colorimetric analyses. Thus, the analyses in Table 4 (columns 1 and 2) suggest real differences (in part) in silica concentrations, depending on the type of water treatment. One should note that below 8 ppm the spectrometric analyses are approaching detection limits, and probably have a scatter of 20 per cent or even more. The sediments squeezed in the laboratory give silicon values which are in fair agreement with those squeezed on board ship, especially for the larger values. The sharp increases in interstitial silica in the presence of abundant amorphous silica, as in Holes 29 and 31 are noteworthy.

\section{DISCUSSION}

The ability of most clay minerals, especially montmorillonite, to take up magnesium and other elements from sea water by cation exchange has been well documented since the experiments of Kelley and Liebig (1934) (see also Carroll and Starkey, 1960; and Keller, 1963). The uptake of magnesium has been especially well documented in studies of interstitial waters from piston cores of oceanic sediments (Shishkina, 1966, and references cited therein). However, the situation for 
TABLE 4

Silica in Pore Waters ${ }^{a}$

\begin{tabular}{|c|c|c|c|c|c|c|c|c|}
\hline Hole & Core & Section & Depth & Description & Age & $\mathrm{Si}(\text { total })^{\mathrm{a}}$ & $\operatorname{Si}(\operatorname{col} .)^{b}$ & $\mathrm{Si}(\mathrm{col} .)^{\mathrm{c}}$ \\
\hline \multirow[t]{3}{*}{23} & 1 & 1 & 2 & Clay, olive gray & Pleistocene & 8.9 & & \\
\hline & 3 & 2 & 66 & “Red clay" silty, zeolitic, Mn. pellets & Miocene & 10.0 & 4.3 & \\
\hline & 4 & 3 & 120 & Clay, olive-green, $\mathrm{Mn}$. & L. Miocene & 10.0 & & \\
\hline \multirow[t]{2}{*}{24} & 2 & 1 & 207 & Clay, gray-green, silty-sandy, glauconite & L. Miocene & 6.8 & & \\
\hline & 4 & 3 & 233 & Clay-mudstone, glauconite, zeolite & L. Miocene & 8.2 & & \\
\hline $24 \mathrm{~A}$ & 3 & 1 & 542 & Graywacke, dark brown & Paleocene-Cret. & 16.0 & 15.0 & \\
\hline \multirow[t]{3}{*}{26} & 1 & 3 & 103 & Clay, dark olive, silty & Pleistocene & 12.0 & 7.3 & 1.5 \\
\hline & 3 & 2 & 230 & Clay, olive-gray, silty & Pleistocene & 7.9 & & \\
\hline & 5 & 3 & 482 & Clay, olive-gray, calcareous & Pleistocene & 7.2 & & \\
\hline \multirow[t]{4}{*}{27} & 2 & 3 & 147 & Clay, gray, silty, sandy, glauconite, pyrite & Miocene & 14.0 & 5.3 & \\
\hline & 3 & 2 & 239 & Clay, olive brown, glauconite, pyrite & Miocene & 15.0 & 3.2 & \\
\hline & 4 & 1 & 246 & Clay, olive brown, glauconite, pyrite & Miocene & 7.5 & 5.5 & \\
\hline & 5 & 2 & 374 & Clay, gray calcareous & Oligocene & 6.7 & & \\
\hline \multirow[t]{4}{*}{$27 \mathrm{~A}$} & 1 & 5 & 34 & Clay, brown, sticky, non-calcareous & Pleistocene & 8.7 & 3.4 & 5.4 \\
\hline & 2 & 3 & 49 & Clay, gray-brown, plastic & Pleistocene & 7.5 & 4.3 & 2.8 \\
\hline & 3 & 3 & 58 & Clay, brown, plastic & Pleistocene & 8.0 & 3.3 & \\
\hline & 4 & 3 & 66 & Clay, brown, plastic & Pleistocene & 7.5 & 2.3 & 2.1 \\
\hline \multirow[t]{2}{*}{28} & 2 & 3 & 77 & Clay, varicolored & $?$ & 14.0 & 12.9 & \\
\hline & 3 & 1 & 171 & Ooze, nannofossil & Eocene & 17.0 & 18.0 & 19.0 \\
\hline \multirow[t]{6}{*}{29} & 1 & 3 & 8 & Clay-ooze, gray-green & Pleistocene & 6.5 & 4.1 & \\
\hline & 4 & 3 & 35 & Clay-ooze, olive & Pliocene & 7.5 & 4.0 & \\
\hline & 9 & 2 & 140 & Radiolarian ooze + chert & M. Eocene & 26.0 & 32.0 & 27.0 \\
\hline & 12 & 6 & $(171)$ & Radiolarian ooze & M. Eocene & 28.0 & 33.0 & 28.0 \\
\hline & 14 & 3 & 192 & Radiolarian ooze & M. Eocene & 28.0 & 33.0 & 28.0 \\
\hline & 17 & 1 & 213 & Radiolarite, cherty dolomite & M. Eocene & 26.0 & 33.0 & \\
\hline
\end{tabular}


TABLE 4 - Continued

\begin{tabular}{|c|c|c|c|c|c|c|c|c|}
\hline Hole & Core & Section & Depth & Description & Age & $\mathrm{Si}\left(\right.$ total) ${ }^{\mathrm{a}}$ & $\mathrm{Si}(\text { col. })^{\mathrm{b}}$ & $\mathrm{Si}(\mathrm{col} .)^{\mathrm{c}}$ \\
\hline \multirow[t]{4}{*}{$29 B$} & 1 & 6 & 65 & Clay, brown & M. Miocene & 9.5 & 6.2 & \\
\hline & 4 & 4 & 96 & Clay, brown & M. Miocene & 9.7 & 7.4 & \\
\hline & 8 & 2 & 126 & Radiolarian ooze + pumice & U. Eocene & $(7.5)$ & 35.0 & \\
\hline & 9 & 4 & 225 & Radiolarian ooze + pumice & M. Eocene & $(8.5)$ & 41.0 & \\
\hline \multirow[t]{6}{*}{30} & 2 & 4 & 67 & Clay-ooze, gray-green & R.-Pleist. & 12.0 & 7.5 & \\
\hline & 3 & 3 & 115 & Clay-ooze, gray-green & R.-Pleist. & 15.0 & 12.9 & \\
\hline & 6 & 2 & 176 & Siltstone, clayey & Pleistocene & 9.0 & & \\
\hline & 11 & 2 & 388 & Siltstone, clayey & M. Miocene & 9.5 & & \\
\hline & 13 & 1 & 403 & Siltstone, clayey & M. Miocene & 21.0 & 21.0 & \\
\hline & 15 & 4 & 419 & Siltstone, clayey & M. Miocene & 10.0 & 7.1 & 4.2 \\
\hline \multirow[t]{3}{*}{31} & 3 & 6 & 68 & Marl, gray & U. Pliocene & 8.5 & 4.5 & \\
\hline & 9 & 2 & 216 & Chalk, light gray & L. Miocene & 26.5 & 23.0 & 19.2 \\
\hline & 10 & 2 & 274 & Chalk, nannoplankton compact & L. Miocene & 30.0 & 33.0 & \\
\hline
\end{tabular}

${ }^{\mathrm{a}} \mathrm{Si}$ (total) is determined by emission spectroscopy (spark) on shipboard-squeezed waters.

${ }^{b}$ Refers to colorimetric determinations on shipboard-squeezed waters.

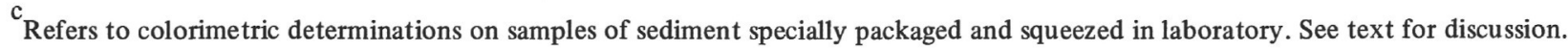


potassium and calcium is more equivocal and controversial. Mangelsdorf et al., (1969) pointed out that potassium appeared to be enriched in interstitial waters of surficial deep ocean sediments by about 10 per cent. They showed that a corresponding diffusive flux of potassium to the overlying water would supply several times more potassium to the oceans than the total supply from rivers. Hence, either the data are misleading or there must exist some compensating sink for potassium, in order to maintain existing oceanic values. One potential source of error in the potassium values has already been referred to, namely, the apparent enrichment in interstitial potassium due to the artificial raising of temperature of the sediments prior to squeezing. The effect is rapid and reversible, and is assumed to be due to a strong and selective temperature influence on the cation exchange capacity.

Actual potassium values in pelagic sediments in Legs 1 to 4 appear to provide indications of modest true enrichments even if maximum corrections for the thermal change are applied-but, as a rule, only in the uppermost few tens of meters. The apparent enrichment due to the temperature effect should decrease with depth because of the natural thermal gradient; that is, assuming normal gradients in sedimentary rocks, room temperature levels should be approached at depths of about one-half kilometer in the sediments. The fact that the enrichments of potassium in pore waters decrease long before this suggests that although temporary enrichments may result from devitrification of glass or feldspar reactions (Correns and Von Engelhardt, 1938), they are balanced in course of time by uptake of potassium by clay minerals (Powers, 1959).

In contrast to the pelagic deposits, potassium is drastically depleted in the more rapidly deposited terrigenous sediments, such as, in Hole 26; and, one must assume a net loss of potassium from bottom waters to the sediments by diffusive flux. Such deficiencies have been previously observed in sediments from the Gulf of Mexico (Manheim and Bischoff, 1968; Manheim, Sayles, and Friedman, 1969). Thus, even if there is an (modified) excess of potassium in surficial pelagic sediments, a compensating loss of potassium takes place in the deep geosynclinal pockets of sediments surrounding the continents. As more interstitial water data are obtained in the nearshore regions, it may become possible to estimate approximately the mass balance.

The calcium relationships involve not only clay-water reactions, but also carbonate equilibria. The authors see clearly both drastic depletions and enrichments of calcium in the interstitial waters, but at this date it has not been possible to distinguish the relative roles of silicate reconstitution (Siever, 1968) and carbonate dissolution or cementation.
Finally, the authors do not yet have a clear answer to why depletions are so great in the "terrigenous" deposits in comparison with the pelagic ones. Are the differences inherent in the nature of the clays or other constituents of the sediments, or is it merely a function of the more rapid sedimentation rate of the terrigenous clays? They are hoping to grapple further with these problems as the water studies for later legs are completed.

\section{ACKNOWLEDGEMENTS}

The authors acknowledge the valuable contributions of D. Kerr, of the Institute of Marine Science, University of Miami, and W. Sunda, of Woods Hole Oceanographic Institution, toward the currently reported work, as well as, the help of Charlotte Lawson and Heidi Richards. Also thanked are D. Spencer, P. Brewer and Roy Smith for placing atomic absorption equipment at the authors' disposal and providing assistance.

\section{REFERENCES}

Behrens, E. W., 1965. Use of the Goldberg refractometer as a salmometer for biological and geological field work. J. Marine Res. 23, 165.

Bischoff, J. L., Greer, R. E. and Luistro, (in press) 1970. Potassium enrichments in interstitial waters of marine sediments: evaluation of temperature of squeezing effect. Science.

Carroll, D. and Starkey, H. C., 1960. Effect of seawater on clay minerals. Clays and Clay Minerals. 7th Conf., London-New York (Pergamon Press) 80.

Correns, C. W. and Von Engelhardt, W., 1938. Neue Untersuchungen uber die Verwitterung des Kalifeldspates. Chem. Erde. 12, 1.

Keller, W. D., 1963. Diagenesis in clay minerals-a review. Clays and Clay Minerals. 11th Conf., LondonNew York (Pergamon Press) 136.

Kelley, W. P. and Liebig, G. F., Jr., 1934. Base exchange in relation to composition of clay with special reference to effect of sea water. Bull. Am. Assoc. Petrol. Geologists. 18, 358.

Mangelsdorf, P. C., Wilson, T. R. S. and Daniel, Ellen, 1969. Potassium enrichments in interstitial waters of recent marine sediments. Science. 165, 171.

Manheim, F. T., 1966. A hydraulic squeezer for obtaining interstitial water from consolidated and unconsolidated sediments. U. S. Geol. Surv. Profess. Paper. 550-C, 256.

Manheim, F. T. and Bischoff, J. L., 1968. Geochemistry of pore waters from Shell Oil Company drill holes on the continental slope of the northern Gulf of Mexico. Chem. Geol. 4, 63.

Manheim, F. T., Sayles, F. L. and Friedman, I., 1969. Interstitial water studies on small core samples, Leg 1, Deep Sea Drilling Project. In Ewing, M. et al., 1970. Initial Reports of the Deep Sea Drilling Project, Volume 1. Washington (U.S. Government Printing Office), 403. 
Muromtsev, A. M., 1963. Osnovnye cherty gidrologii Atlanticheskogo okeana. Prilozheniye 2, Atlas vertikaltnykh razrezov $i$ kart temperatury, solensoti, plotnosti $i$ soderzhaniya kisloroda (Basic features of hydrology of the Atlantic Ocean, Appendix 2, Atlas of vertical sections and maps of temperature, salinity, density and oxygen content). Gidrometeorologischeskoye izdatel'stvo, Moscow, 183.

Powers, M. C., 1959. Adjustment of clays to chemical change and the concept of the equivalence level. Clays and Clay Minerals. 6th National Conf., New York (Pergamon Press) 309.
Shishkina, O. V., 1966. Osnovnoi sostav (General chemical composition). In Khimiya Tikhogo okeana. S. W. Brujewicz (Ed.). Ch. 2, Khimiya gruntovykh rastvorov Tikhogo okeano (Chemistry of the Pacific Ocean, pt. 2, Chemistry of pore waters in the Pacific Ocean), 289-307, combined references, 342358.

Siever, Raymond, 1968. Sedimentological consequences of a steady-state ocean-atmosphere. Sedimentology. 11,5 . 